\title{
Nuclear clustering in processes of relativistic multifragmentation *
}

V. Bradnova ${ }^{\mathrm{a}}$, M. M. Chernyavsky ${ }^{\mathrm{b}}$, A. Sh. Gaitinov ${ }^{\mathrm{c}}$, L. A. Goncharova ${ }^{\mathrm{b}}$, L. Just ${ }^{\mathrm{d}}$, S. P. Kharlamov ${ }^{b}$, A. D. Kovalenko ${ }^{a}$, M. Haiduc ${ }^{\text {, }, ~ V . ~ G . ~ L a r i o n o v a ~}{ }^{\text {b }}$, F. G. Lepekhin ${ }^{\mathrm{f}}$, A. I. Malakhov ${ }^{a}$, G. I. Orlova ${ }^{\mathrm{b}}$, N. G. Peresadko ${ }^{\text {b }}$, N. G. Polukhina ${ }^{\mathrm{b}}$, P. A. Rukoyatkin ${ }^{\mathrm{a}}$, V. V. Rusakova ${ }^{a}$, N. A. Salmanova ${ }^{\mathrm{b}}$, B. B. Simonov ${ }^{\mathrm{f}}$, S. Vokal, P. I. Zarubin ${ }^{\mathrm{a}}$,

I. G. Zarubina ${ }^{a}$

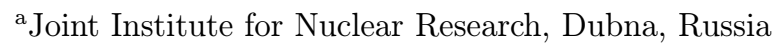

${ }^{b}$ P. N. Lebedev Physical Institute, Moscow, Russia

'Institute of Physics and Technology, Almaty, Kazakstan

dP. J. Šafárik University, Košice, Slovakia

eInstitute of Space Sciences, Bucharest-Maguerel, Romania

${ }^{\mathrm{f}}$ Petersburg Institute of Nuclear Physics, Gatchina, Russia

Unique capabilities of emulsion technique are considered as applied to the study of multifragmentation of light stable and radioactive nuclei in peripheral interactions at beam energy of few $\mathrm{GeV}$ per nucleon. The importance of this research for the physics of few body nuclear systems and the related problems of nucleosynthesis is noted. New results on dissociation of ${ }^{7} \mathrm{Be}$ and ${ }^{22} \mathrm{Ne}$ in very peripheral interactions with emulsion nuclei are presented as an illustration.

\section{Introduction}

The BECQUEREL Project (Beryllium (Boron) Clustering Quest in Relativistic Multifragmentation) is oriented toward emulsion expositions by light stable and radioactive nuclei with an energy of the order of few $\mathrm{GeV}$ per nucleon in the JINR Nuclotron beams. Observations of the fragmentation of light relativistic nuclei open up new opportunities to explore highly excited nuclear states near multiparticle decay thresholds [1]. Our interest in such states is motivated by their predicted properties as loosely bound systems with spatial spread significantly exceeding the fragment sizes. Natural components of such states are lightest nuclei having no excited states below particle decay thresholds, i. e. deuterons, tritons, ${ }^{3} \mathrm{He}$, and ${ }^{4} \mathrm{He}$ nuclei.

\footnotetext{
*This work has been supported by the grants 96-15-96423, 03-02-16134, 02-02-164-12a of the Russian foundation of basic researches, VEGA N1/9036/02 of the Agency of Science of the Ministry of Education of the Slovak Republic and the Slovak Academy of Sciences, and the grants of the JINR Plenipotentiaries of Slovakia and Romania in the years 2002 and 2003.
} 


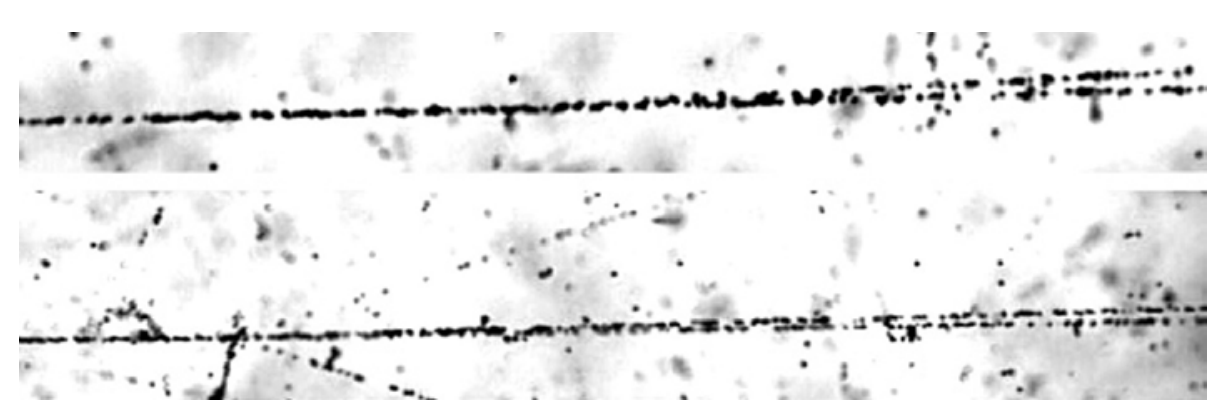

Figure 1. Examples of peripheral dissociation of $1.23 \mathrm{~A} \mathrm{GeV}{ }^{7} \mathrm{Be}$ nuclei into pairs of He nuclei. Upper photo: dissociation without target nucleus excitation and produced charged mesons. Lower photo: dissociation accompanied by a target fragment and a meson like pair.

A complete spectroscopy of few body decays of highly excited nuclei allows one to search for the Efimov excited states in nuclear systems [2,3]. The Efimov effect is the remarkable prediction that numbers of bound states for three particles interacting via s-wave short range potentials may grow to infinity, as the pair interaction is just about to bind two particles. The Efimov states are loosely bound and their wave functions extend far beyond those of the remaining two particles.

Other intriguing conjecture is that $\mathrm{n} \alpha$ nuclei near the $\mathrm{n} \alpha$ particle decay threshold can constitute a loosely bound dilute gas forming a Bose condensate [4]. Its major signature is a multiple $\alpha$ particle decay with a narrowed distribution of relative velocities. Search for such states on the nuclear scale is of undoubted interest since they can play a role of intermediate states in a stellar nuclear fusion due to reduced Coulomb repulsion.

In this respect, an experimental task consists in the provision of a complete spectroscopy of final fragments, i. e. observation of dissociation, determination of various channel probabilities, fragment identification and velocity measurement. Our approach is based on spectroscopy of relativistic fragments of projectile nuclei at longitudinal exposures of emulsion layers.

Advantages of an emulsion technique are exploited most completely in the study of peripheral fragmentation of light stable and neutron deficient nuclei. Visual scanning is concentrated on the events with a total charge transfer of an incoming nucleus to secondary particles in a narrow fragmentation cone. Emulsion nucleus fragmentation and meson production become reduced or even suppressed in this way. Such events amount to a few percent of the total number of inelastic events. In practice, this approach allows one to accumulate statistics of a few tens of peripheral events, which is sufficient for a reliable determination of dominating dissociation channels. Earlier, a dominating role of channels ${ }^{6} \mathrm{Li} \rightarrow \alpha \mathrm{d}[5,6],{ }^{12} \mathrm{C} \rightarrow \alpha \alpha \alpha[7]$ was established. At present an analogous analysis of ${ }^{14} \mathrm{~N},{ }^{7} \mathrm{Li}$ and ${ }^{11} \mathrm{~B}$ exposures is carried out in order to find effects of a deuteron and triton clustering. Our first results for ${ }^{7} \mathrm{Be}$ and ${ }^{22} \mathrm{Ne}$ nuclei are discussed in what follows. 


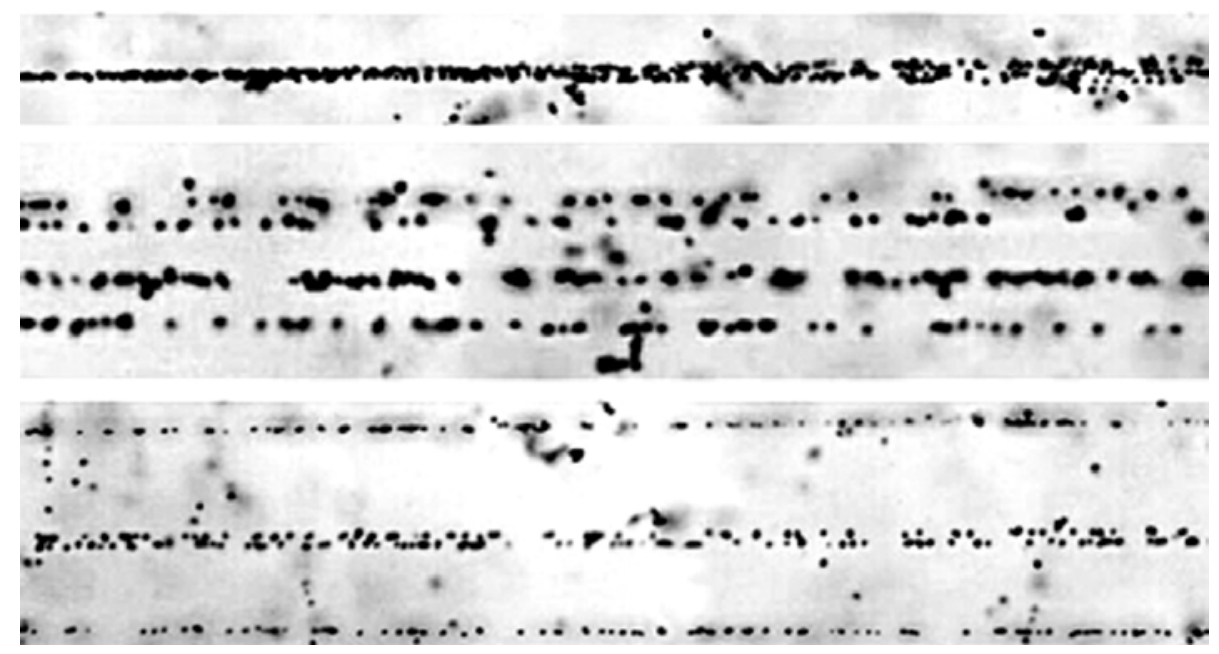

Figure 2. Dissociation of $4.5 \mathrm{~A} \mathrm{GeV} / \mathrm{c}$ Ne nucleus in peripheral interaction into five He fragments. Upper photo: Interaction vertex with production of narrow fragment jet. Middle photo: shifting from vertex allow one to identify three He fragments. Lower photo: further shifting allow to resolve a central track on the previous photo as a very narrow pair of relativistic He nuclei ( ${ }^{8}$ Be production).

\section{Clustering in ${ }^{7} \mathrm{Be}$ dissociation}

A secondary beam containing a significant fraction of $1.23 \mathrm{~A} \mathrm{GeV}{ }^{7} \mathrm{Be}$ nuclei was formed at the JINR Nuclotron by selecting the products of charge exchange of primary ${ }^{7} \mathrm{Li}$ nuclei with aid one of the beam transport channels. Emulsion stacks have been irradiated. The ${ }^{7} \mathrm{Be}$ nucleus is convenient for magnet optics selection due to the maximum ratio of the charge to the weight. Besides, it gives most complete observation of final fragments.

By visual scanning along tracks, we have found 22 decays of incoming nuclei to helium fragments without other accompanying tracks ("white stars"). The event examples are shown in the Figure 1. Helium isotopes have been identified via their total moments derived from multiple scattering measurements. This is allowed to conclude, that a dominant fraction is due to a coherent dissociation ${ }^{3} \mathrm{He}+{ }^{4} \mathrm{He}$ and only $2-3$ decays to ${ }^{3} \mathrm{He}+{ }^{3} \mathrm{He}+\mathrm{n}$. We have found 20 events with charged topology of relativistic fragments $2+1+1$ ("white stars") with identified ${ }^{3} \mathrm{He}$ and 16 with identified ${ }^{4} \mathrm{He}$. The events with topology $3+1$ and $1+1+1+1$ are under analysis now. They represent only few percent fraction.

Thus, one can conclude that a ${ }^{3} \mathrm{He}$ clustering manifests in decays of excited relativistic ${ }^{7} \mathrm{Be}$ nuclei. This result agrees with our expectations. It seems to be useful for future studies of the role of ${ }^{3} \mathrm{He}$ clusterization in decays ${ }^{8} \mathrm{~B}\left({ }^{1,2} \mathrm{H}-4,{ }^{4} \mathrm{He}-{ }^{3} \mathrm{He}\right),{ }^{9} \mathrm{C}\left({ }^{3} \mathrm{He}-{ }^{3} \mathrm{He}^{3} \mathrm{He}\right)$, ${ }^{10} \mathrm{C}\left({ }^{3} \mathrm{He}-{ }^{3} \mathrm{He}-{ }^{4} \mathrm{He}\right)$, and ${ }^{11} \mathrm{C}\left({ }^{3} \mathrm{He}-{ }^{4} \mathrm{He}-{ }^{4} \mathrm{He}\right)$. It is quite possible that ${ }^{3} \mathrm{He}$ clustering could play a role analogous to the role of a triple $\alpha$ process in stellar nucleosynthesis. 


\section{Coherent dissociation of ${ }^{22} \mathrm{Ne}$ nuclei}

Universality of a coherent dissociation mechanism enables us to search for such events in emulsions irradiated by ${ }^{24} \mathrm{Mg},{ }^{28} \mathrm{Si}$, and ${ }^{32} \mathrm{~S}$ nuclei at $4.5 \mathrm{~A} \mathrm{GeV} / \mathrm{c}$ in order to study a relative role of multiparticle decays. As a first step we reanalyzed of existing data (DST) on about $4100{ }^{22} \mathrm{Ne} 4.1 \mathrm{~A} \mathrm{GeV} / \mathrm{c}$ interactions in an emulsion. We found 94 events containing only fragments of a primary nucleus without target nucleus fragments and produced mesons.

The dominant channel is $8+2-53$ events (He cluster separation), the follow $9+1-$ $14,7+2+1-7,8+1+1-6,6+2+2-5,6+2+1+1-3$. Among deeper fragmentation there are 2 events $4+1+1+1+1+1+1$ and $4+2+2+2$, and single events with topologies $5+2+1+1+1,5+2+2+1,3+2+1+1+1+1+12+2+2+2+1+1$. The most interesting fact is that we have found 3 events of topology $2+2+2+2+2$, corresponding neon dissociation into helium nucleus fragments only. The example is shown in the Figure 2.

In our opinion, such distribution of final charged states brightly illustrate transition from a single helium fragment splitting to a total multifragmentation of the explored nucleus. Absence in the selected statistics of binary fissions like $7+3,6+4,5+5$ is especially interesting. We plan to carry out a detailed kinematical analysis of these events. Verification of a hypothesis about the phase transition of light nuclei from a ground state to multiparticle one via a Bose condensate is one of intriguing perspectives of this research.

\section{REFERENCES}

1. V. Bradnova et al. Few-Body Systems Suppl. 14 (2003) 241.

2. V. Efimov Phys. Lett. B33, 563 (1970).

3. F. Nunes C. R. Physique 4 (2003) 489-485

4. P. Schuck, H. Horiuchi, G. Ropke, A. Tohsaki, C. R. Physique 4 (2003) 537-540.

5. M. I. Adamovich et al. Phys. At. Nucl., vol.. 62, N8, 1999, pp 1378-1387.

6. F. G. Lepekhin, D. M. Seliverstov, B. B. Simonov. Eur. Phys. J. A, 1, 137-141 (1998).

7. V. V. Belaga et al. Phys. Atom. Nucl., vol. 58, c. 2014-2020,1995. 